\title{
sciendo
}

\section{Post-Activation Potentiation: Is there an Optimal Training Volume and Intensity to Induce Improvements in Vertical Jump Ability in Highly-Trained Subjects?}

\author{
by \\ Ronaldo Kobal ${ }^{1}$, Lucas A. Pereira ${ }^{1}$, Katia Kitamura ${ }^{1}$, Anderson C. Paulo ${ }^{2}$, \\ Henrique A. Ramos ${ }^{3}$, Everton C. Carmo ${ }^{4}$, Hamilton Roschel ${ }^{3}$, Valmor Tricoli’, \\ Chris Bishop ${ }^{5}$, Irineu Loturco ${ }^{1,6}$
}

The aim of this study was to compare the acute effects of performing half squats (HSs) with different loading intensities (1,3, and 5 repetitions maximum [RM], and 60\% 1RM) and a different number of sets $(1,2$, and 3$)$ on the countermovement jump (CMJ) performance of 18 highly-trained male subjects. Participants were submitted to four experimental conditions (1RM, 3RM, 5RM, and 60\% 1RM) in randomized order. The CMJ was assessed before and after each set. Differences in CMJ performance between the distinct experimental conditions and individual responses in CMJ performance induced by the different protocols were analyzed via the magnitude-based inference method. Overall, significant improvements were detected in individual CMJ heights after each activation protocol. It can be concluded that the use of 1 to 3 sets of HSs performed at moderate-to-high loads may be an effective strategy to improve jump performance in highly-trained subjects. Nevertheless, despite the high efficiency of the protocols tested here, coaches and researchers are strongly encouraged to perform individualized assessments within the proposed range of loads and sets, to find optimal and tailored post-activation potentiation protocols.

Key words: warm-up, vertical jump, muscle power, conditioning activity, athletic performance, strength training.

\section{Introduction}

Strength and power abilities play a key role in many competitive sports (Cormie et al., 2011a, 2011b; Cronin and Sleivert, 2005). Therefore, coaches and researchers are constantly searching for novel and more effective approaches to improve these capacities. It has been proposed that the use of resistance exercises with moderate or high loads during athletes' warm-up routines, might work as a type of conditioning activity (CA), possibly inducing meaningful enhancements in subsequent motor-tasks (Ebben, 2006; Hodgson et al., 2005; Sale, 2002). This phenomenon is known as postactivation potentiation (PAP), and it seems to be attributed to specific physiological and neuromuscular responses, such as increased phosphorylation of the regulatory light-chains of myosin and increased recruitment rates of motor units (Ebben, 2006; Hodgson et al., 2005; Rassier and Macintosh, 2000; Sale, 2002). From these effects, significant increases in muscle tension and, consequently, in strength and power production have been observed (Rassier and Macintosh, 2000; Tillin and Bishop, 2009).

1 - NAR - Nucleus of High Performance in Sport, São Paulo, Brazil.

2 - Academic Department of Physical Education of the Federal Technological University of Paraná, Brazil.

3 - School of Physical Education and Sport, University of São Paulo, São Paulo, Brazil.

4 - Physical Education, Senac University Center, São Paulo, Brazil.

5 - Faculty of Science and Technology, London Sports Institute, Middlesex University, London, UK.

6 - Department of Human Movement Sciences, Federal University of São Paulo, São Paulo, Brazil. 
Acute increases in performance can be substantially affected by the balance between PAP mechanisms and fatigue (Chatzopoulos et al., 2007; Jones and Lees, 2003; Scott and Docherty, 2004; Young et al., 1998). These "opposing effects" have usually produced inconsistent findings and unclear training guidelines (Lowery et al., 2012; Mola et al., 2014; Sale, 2002). Moreover, it appears that this balance may be altered by several factors, such as training experience, length of the rest period prior to subsequent exercise, and volume and intensity of CA (Wilson et al., 2013). For this reason, many studies have been designed to determine the optimal PAP strategy to induce positive short-term effects in trained subjects (Gourgoulis et al., 2003; Hanson et al., 2007; Jones and Lees, 2003; Young et al., 1998). On the whole, these investigations have adopted different intensities and volumes of CA, triggering distinct neuromuscular responses in subsequent poweractivities (i.e., vertical jump and sprint performance) (Bevan et al., 2010; Chatzopoulos et al., 2007; Gourgoulis et al., 2003; Jones and Lees, 2003; Linder et al., 2010). Young et al. (1998) demonstrated an increase of $2.8 \%$ in countermovement jump (CMJ) height after 1 set of 5 repetitions maximum ( $\mathrm{RM}$ ) in the half-squat (HS) exercise. Gourgoulis et al. (2003) found increases of $2.39 \%$ in CMJ height after applying a progressive HS protocol (1 set of 2 repetitions), using a range of loads varying from 20 to $90 \%$ 1RM. Similarly, Smilios et al. (2005) investigated the effects of 3 sets of 5 repetitions of the HS at $60 \% 1 \mathrm{RM}$ on vertical jump performance, reporting significant increases of 3.74 and $2.84 \%$ after the first and second exercise sets (respectively, compared with the pre-values). Conversely, Jones and Lees (2003) observed no changes in CMJ height after executing a single set of 5 repetitions of the HS at $85 \% 1 \mathrm{RM}$. In another study with comparable outcomes, Hanson et al. (2007) reported no enhancements in vertical jump height after using different CA strategies (i.e., "lowintensity protocol": 8 repetitions of the HS at $40 \%$ 1RM and "high-intensity protocol": 4 repetitions of $\mathrm{HS}$ at $80 \% 1 \mathrm{RM})$. Collectively, these findings suggest that there is a notable lack of consensus about the best way to properly induce PAP.

In an attempt to clarify this issue, Wilson et al. (2013) reported in their meta-analysis an optimal range of training content to optimize PAP effects, suggesting the use of multiple sets (vs single), moderate loading intensity (i.e., from 60 to $85 \% 1 \mathrm{RM})$, and rest periods lasting between 7 and 10 minutes. However, these ranges may vary across individuals, according to their training status. Although these general guidelines allow sport practitioners to design better CA protocols, more information on this topic is needed. Thus, the objective of this study was to compare the effects of different loading intensities $(1,3,5 \mathrm{RM}$, and $60 \% 1 \mathrm{RM})$ and numbers of sets $(1,2$, and 3$)$ of the HS on the subsequent CMJ performance in highly-trained subjects (i.e., subjects able to lift at least $2 x$ their body-mass (BM) in the HS). Due to the conflicting results in the literature and considering previous findings related to this topic (Crewther et al., 2011; Ruben et al., 2010; Seitz et al., 2014), we hypothesized that the participants would attain significant increases in jump height, presenting their best performances at different ranges of intensities and volumes.

\section{Methods}

\section{Study Design}

To test the influence of HS conditioning activities with different loading intensities (1, 3, and $5 \mathrm{RM}$, and $60 \% 1 \mathrm{RM}$ ) and a different number of sets $(1,2$, and 3$)$ to induce PAP on the subsequent CMJ performance, participants were submitted to four experimental sessions. A control session with jump tests was conducted to observe if this procedure could induce PAP. In this session, participants underwent CMJ assessments with the same rest intervals used throughout the experimental sessions. Prior to the experimental sessions, participants performed 5 familiarization sessions comprising all procedures of the study, which were also used to determine the 1,3 , and 5RM loads, all separated by at least 72 hours.

After the familiarization and load determination sessions, participants were submitted to four experimental conditions (1RM, $3 R M, 5 R M$, and $60 \% 1 R M$ ) in randomized order. Before the experimental sessions, participants performed a general warm-up, which consisted of running on a treadmill at $9.0 \mathrm{~km} \cdot \mathrm{h}^{-1}$ for $5 \mathrm{~min}$ followed by $3 \mathrm{~min}$ of dynamic stretching and submaximal CMJ attempts. Subsequently, the preCMJ assessments were carried out. After a 4-min rest interval, a specific warm-up was performed (5 repetitions with $50 \%$ of the CA load of the day). 
Three minutes later the first set of the respective CA was performed, with participants instructed to execute each repetition as fast as possible. For the $60 \% 1 \mathrm{RM}$ condition, participants performed 6 repetitions. After a 4-min rest interval, participants repeated the CMJ test. This procedure was repeated three times. A schematic presentation of the study design is demonstrated in Figure 1.

\section{Participants}

Eighteen highly-trained men (e.g., college athletes from different sports, with at least 3 years of systematic resistance-training experience) able to lift at least two-times their body mass in the HS exercise participated in the study (age: $25.42 \pm 3.58$ years; body height: $175.17 \pm 7.35 \mathrm{~cm}$; body mass: $78.84 \pm 10.63 \mathrm{~kg}$ ). This inclusion criterion was selected based on previous studies, suggesting an adequate strength level necessary to induce PAP (Chiu et al., 2003; Seitz et al., 2014). All participants were free from any musculoskeletal injuries. They were asked to refrain from strenuous physical activities and caffeine consumption 24 hours prior to the experimental sessions. Before participating in this study, the athletes were briefed on the experimental design and signed an informed consent form. This study was performed in accordance with the ethical standards of the Helsinki Declaration and was approved by the local ethics committee (Research Ethics Committee, CEP-EEFEUSP).

\section{Determination of the 1, 3, and 5RM loads in the half-squat exercise}

After a general warm-up, participants performed two specific warm-up sets for each test. In the first set, they performed 5 (for 1 and 3RM tests) or 10 repetitions (for 5RM test) with $50 \%$ of the respective estimated load; after a 3-min rest interval, they performed 3 (for 1 and 3RM tests) or 5 (for 5 RM test) repetitions with $70 \%$ of the estimated load for each test (Brown and Weir, 2001). Three minutes later, participants were allowed to execute up to 5 attempts to obtain the 1,3 , and 5RM loads in the HS exercise, performed on the Smith-Machine equipment (Nakagym ${ }^{\circledR}$, Diadema, Brazil). A 3-min rest interval was permitted between the following attempts.

\section{Countermovement Jump Test}

Vertical jump height was determined using the CMJ. Participants were instructed to execute a downward movement followed by a complete extension of the legs and were free to determine the countermovement amplitude to avoid possible changes in jumping coordination. The CMJ attempts were executed with the hands on the hips. All jumps were performed on a contact platform (Jump Test 2.0, Hidrofit Ltda, Belo Horizonte, Brazil) and the obtained flighttime $(t)$ was used to estimate jump height (h) (i.e., $\mathrm{h}=\mathrm{gt}^{2} / 8$, where $\left.\mathrm{g}=9.81 \mathrm{~m} \cdot \mathrm{s}^{-1}\right)$. A total of 5 attempts were performed, interspersed with $\sim 15$ s. The best CMJ attempt in each condition was retained for further analysis.

\section{Statistical Analysis}

Data are presented as mean \pm standard deviation (SD). Normality of data was checked using the Shapiro-Wilk test. To analyze the differences in CMJ performance between the different CA protocols across the 3 sets, the differences based on magnitudes were calculated (Batterham and Hopkins, 2006). The quantitative chances of finding differences in the CMJ between the different loading intensities and sets were assessed qualitatively as follows: $<1 \%$, almost certainly not; 1 to $5 \%$, very unlikely; 5 to $25 \%$, unlikely; 25 to $75 \%$, possible; 75 to $95 \%$, likely; 95 to $99 \%$, very likely; and $>99 \%$, almost certain (Batterham and Hopkins, 2006). A meaningful difference was considered using the mechanistic inference, based on threshold chances of $5 \%$ for substantial magnitudes. Thus, if the chances of having better and poorer results were both $>5 \%$, the true difference was assessed as unclear. Additionally, to determine the magnitude of the differences between the different conditions across the three sets for the CMJ height, the standardized differences (ES: Cohen's $d$ ) were calculated (Hopkins et al., 2009). The ES magnitudes were interpreted using the following thresholds: <0.2, $0.2-0.6,0.6-1.2,1.2-2.0,2.0-4.0$, and $>4.0$ for trivial, small, moderate, large, very large, and near perfect, respectively (Hopkins et al., 2009).

As great variation was observed in individual responses in the $\mathrm{CMJ}$ performances induced by different CA protocols across the 3 sets, an individual analysis was further performed using the magnitude-based inference principles. Terms such as possibly and unclear were used if the typical error of measurement (TE; SD $/ \sqrt{2}$ ) crossed one or both smallest worthwhile change (SWC; calculated by using $0.3 \times$ within subject 
coefficient of variation) boundaries (Hopkins, 2004), respectively. Otherwise, if the TE did not cross SWC boundaries, the effect was inferred as probably.

\section{Results}

The absolute loads corresponding to 1RM, $3 R M, 5 R M$, and $60 \% 1 R M$, used for the CA protocols in the HS exercise were: $187.83 \pm 30.02$ $\mathrm{kg} ; 174.89 \pm 31.24 \mathrm{~kg} ; 165.28 \pm 28.48$; and $112.7 \pm$ $18.01 \mathrm{~kg}$, respectively. Figure 2 depicts group comparisons of the different CA protocols across the three sets. After the first set of CA meaningful differences were not observed in CMJ performance when compared with the pre-values. After the second set the control condition demonstrated a likely decrease (percentage chances 81/19/00), while the 5RM condition demonstrated a possible reduction $(65 / 35 / 00)$ in CMJ performance when compared with the prevalues. In the final set, all protocols demonstrated possibly to almost certainly lower $\mathrm{CMJ}$ heights than the pre-values $(81 / 19 / 00,79 / 21 / 00,57 / 43 / 00$, $100 / 00 / 00$, and $80 / 20 / 00$, for control, $1 \mathrm{RM}, 3 R M$, $5 \mathrm{RM}$, and $60 \% 1 \mathrm{RM}$ conditions). No meaningful differences were observed for the changes in CMJ performance between the distinct loading intensities across the 3 sets.

Figure 3 shows individual analyses of the CMJ percentage changes across the different conditions. In the control condition, 2 participants demonstrated meaningful increases in the CMJ height after the $3^{\text {rd }}$ set in comparison to the prevalues. For the 1RM condition, meaningful increases in the CMJ height were observed on 13 distinct occasions for 6 participants when compared to pre-values. In the 3RM protocol, meaningful improvements in $\mathrm{CMJ}$ performance were observed for 5 participants on 11 different occasions. For the 5RM condition, meaningful increases were noted in the CMJ height for 5 participants on 7 different occasions. Finally, for the $60 \% 1 \mathrm{RM}$ protocol meaningful improvements in $\mathrm{CMJ}$ performance were found on 11 distinct occasions for 4 participants.

\section{Discussion}

This study aimed to compare the acute effects of performing the HS under four different experimental conditions, with distinct numbers of sets $(1,2$, and 3$)$ and loading intensities (1RM, $3 \mathrm{RM}$, or $5 \mathrm{RM}$; and at $60 \% 1 \mathrm{RM}$ ) on subsequent vertical jump ability. Overall, we did not find meaningful differences between any of the examined protocols. However, we detected worthwhile improvements in individual performances after each CA protocol, thus observing a wide variation in the training content able to induce acute increases in CMJ height. Therefore, it seems reasonable to assume that the PAP phenomenon may be subject-dependent and lacks optimal standards for both volume and intensity.

As aforementioned, there are conflicting findings among researchers about the best way to induce PAP in trained subjects (Chatzopoulos et al., 2007; Jones and Lees, 2003; Scott and Docherty, 2004; Seitz et al., 2014; Young et al., 1998). Whereas some studies have shown acute improvements in performance (Crewther et al., 2011; Golas et al., 2017; Seitz et al., 2014), others have reported no changes, or even fatigue-related symptoms after the execution of different CA protocols (Lim and Kong, 2013; Scott and Docherty, 2004). For many authors, the absence of consistent findings about PAP is related to a varied number of factors which affect this complex phenomenon, such as the exercise type, the strength level, the training background, and the rest interval between $\mathrm{CA}$ and the subsequent activity (Bevan et al., 2010; Esformes et al., 2011; Fukutani et al., 2014; Wilson et al., 2013). Seitz et al. (2014) found significant relationships between the relative strength index (i.e., $1 \mathrm{RM} / \mathrm{kg}$ ) and the degree of potentiation induced in muscle power $(r$ $=0.65$ ), achieved after 3 repetitions of the squat at $90 \%$ 1RM. Accordingly, Ruben et al. (2010) observed that, after executing an ascending squat protocol (i.e., 5 repetitions at 30\% 1RM, 3 at $70 \%$ $1 \mathrm{RM}$, and 3 at $90 \% 1 \mathrm{RM}$ ), only the stronger individuals (squat $\geq 2.0 \times \mathrm{BM}$ ) exhibited acute enhancements in horizontal jump ability. That said, it seems that as the maximum strength capacity increases, the aptitude to elicit PAP also increases (Golas et al., 2017). Since we selected participants able to lift at least twice their BM in the HS exercise, this is probably the reason why almost all of them (16 of 18) presented significant improvements in jump performance. 


\begin{tabular}{|c|c|}
\hline Familarization and test $s$ & \\
\hline 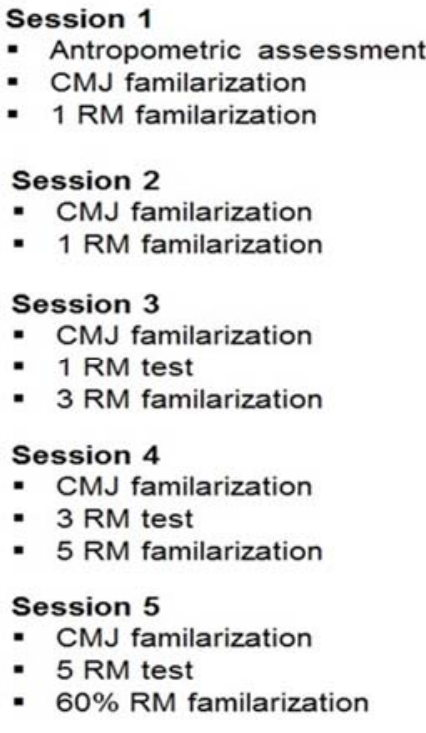 & 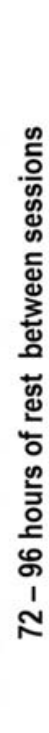 \\
\hline
\end{tabular}

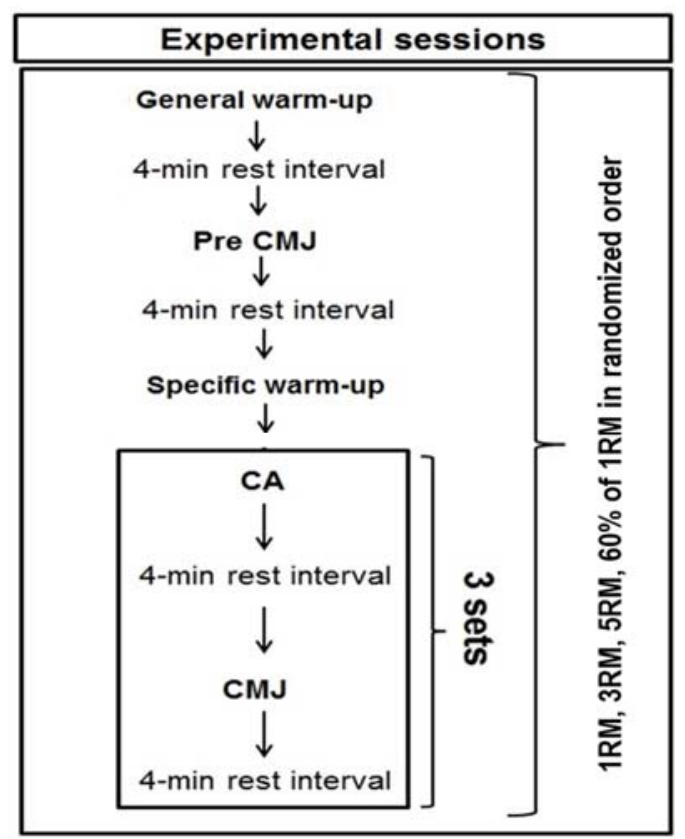

Figure 1

Schematic presentation of the study design.

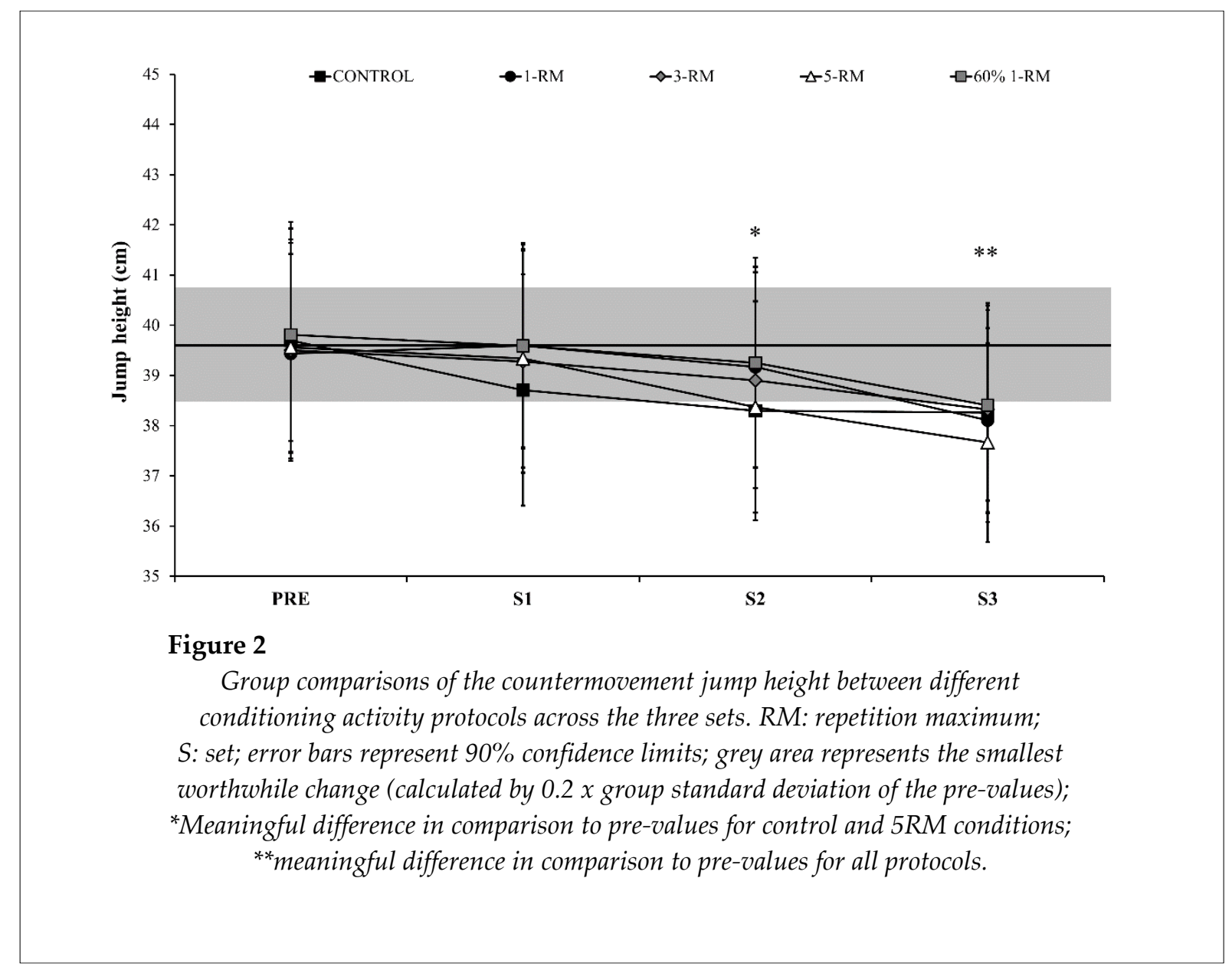



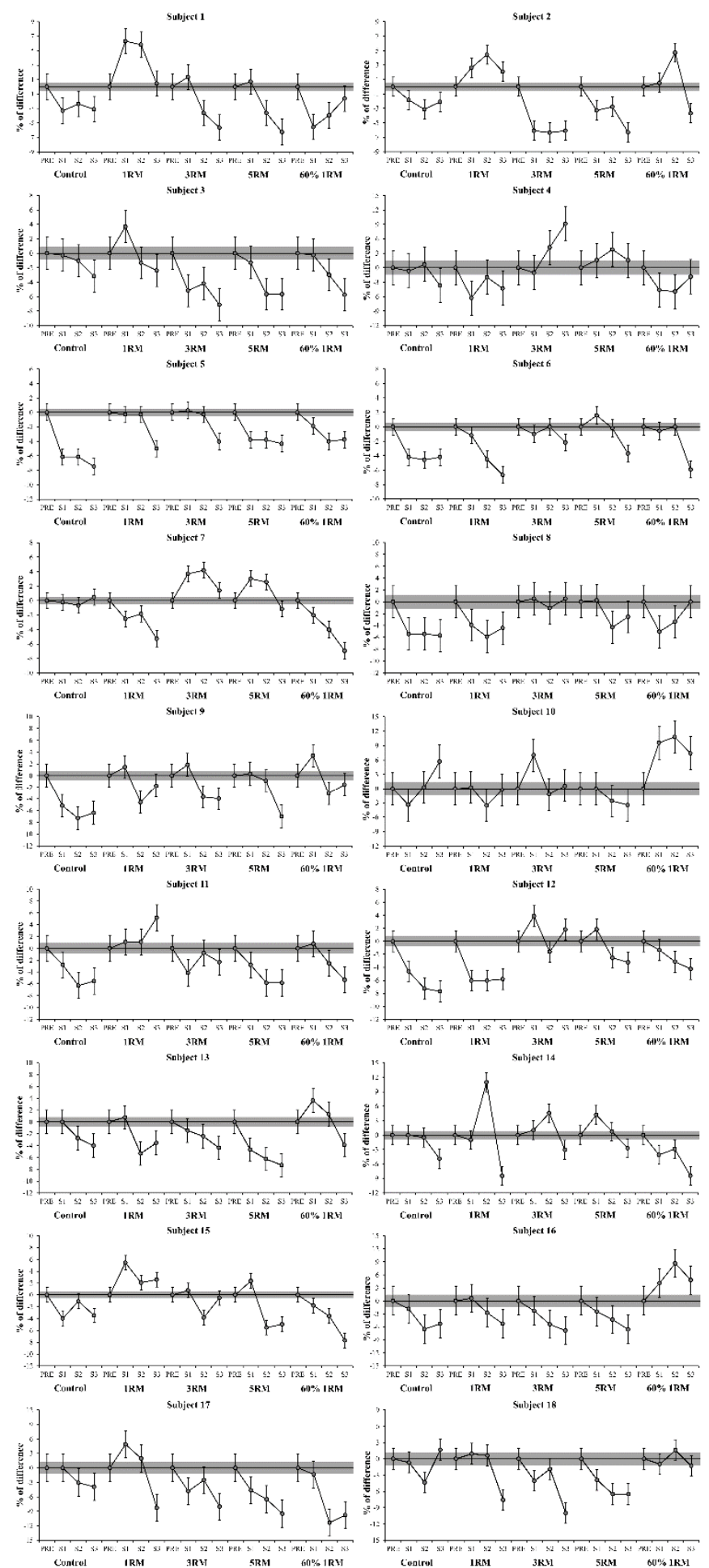

Figure 3

Individual analyses of the countermovement jump percentage change across the different experimental conditions. RM: repetition maximum; S: set; error bars represent typical error of measurement (TE); grey area represents the smallest worthwhile change

(SWC: calculated by $0.3 x$ individual coefficient of variation).

Terms such as possibly and unclear were used if the TE crossed one or both SWC boundaries, respectively. 
Besides the training status, three other important factors were carefully controlled in this study: the rest interval between HS and CMJ tests (4 $\mathrm{min}$ ), the range of loads used to perform the CA (HS at $1 \mathrm{RM}, 3 \mathrm{RM}, 5 \mathrm{RM}$, and $60 \% 1 \mathrm{RM}$ ), and the mixed number of sets among the different conditions. Seitz et al. (2014) suggested that the temporal profile of PAP was related to relative strength levels, with stronger subjects (i.e., individuals able to squat at least twice their BM) achieving maximum PAP responses between the 3rd and 12th minute post-CA. Regarding volume and intensity, a comprehensive meta-analysis indicated that multiple sets (e.g., 2 or 3) and moderate loads (60-84\% 1RM) were more prone to optimize PAP than single sets or heavy loads ( $\geq$ $85 \%$ 1RM) (Wilson et al., 2013). Thus, across the five protocols, our participants could certainly perform the HS under (theoretical) "optimal training conditions"; nevertheless, no CA was superior to another to maximize PAP. Despite the apparent superiority of the 1RM condition, the absence of meaningful differences between the examined protocols does not allow to draw definitive conclusions from our data. Moreover, the multitude of individual best $\mathrm{CMJ}$ responses among sets (Figure 3) also precludes accurate determination of the most effective approaches to activate PAP (e.g., numbers of sets and percentages of 1RM).

In fact, we observed worthwhile increases in CMJ height only when comparing the highest intra-individual $\mathrm{CMJ}$ attempts with their respective baseline tests. From a practical perspective, this suggests that acute PAP effects can possibly occur in highly-trained subjects after conditioning activities; nonetheless, these effects seem to be subject-dependent, depending on specific and individualized training conditions (Figure 3). Remarkably, almost all participants (16 of 18) were responsive to at least one experimental protocol, which strongly indicates that PAP methods might be effectively used by coaches to enhance performance in highly-trained athletes, provided they have previously tested the effects of a given CA in an individualized way. This "apparent" individual variability has also been observed in other PAP studies. Bevan et al. (2010) investigated the effects of performing 1 set of 3 repetitions of the HS at 91\% 1RM with different rest intervals (4-, 8-, 12-, and 16-min) on short sprint performance (5- and 10-m). Whereas the authors failed to find significant differences between conditions, they reported significant improvements in speed ability when the best sprint time of each participant was compared with their respective baseline value. Briefly, the professional rugby players revealed distinct optimal responses to each specific rest interval: $47 \%$ of them were positively responsive after the 8 -min, $27 \%$ after the 12 -min, and $13 \%$ after the 4 or 16-min rest interval. Lim and Kong (2013) detected no significant differences between three different CA protocols which used isometric or dynamic muscle actions after analyzing their effects on maximal sprint velocity in well-trained track athletes. However, in line with our findings, the authors observed large individual variations in response to the PAP schemes, with some athletes benefiting from the acute potentiation effects and others not.

In summary, at least for highly-trained subjects, our data demonstrate that there is no general recommendation for the optimal training content (i.e., volume and intensity) to maximize PAP. Furthermore, since it is probable that stronger athletes may benefit from the inclusion of conditioning activities in their warm-up routines, coaches and researchers are encouraged to frequently assess their individual responses to distinct CA protocols, adjusting or modifying the training content whenever necessary. Certainly, this strategy could improve an individual's athletic performance in many sport disciplines, especially those relying on speed-power capacities. This study is limited by the lack of individualization of resting periods. As demonstrated by Golas et al. (2016), the individualization of rest intervals after the CA would be important to find the "best strategy for each subject". Nonetheless, for such a purpose, it would be necessary to substantially increase the number of experimental sessions during the intervention period, which was not possible in the current study.

\section{Conclusion}

This study may have important implications in high performance sport settings. From our data, it can be concluded that the use of 1 to 3 sets of half squats performed at moderate to high training loads may be a very effective 
strategy to meaningfully improve explosive performance in highly-trained subjects. Nevertheless, despite the high efficiency of the CA conditions tested here, coaches and sport scientists should individually assess their athletes within the proposed range of loads and sets, to find optimal (and tailored) PAP protocols, able to acutely maximize speed and power capacities. To evaluate and quantify individual responses, practitioners can make use of the "smallest worthwhile change", which is defined as the smallest change in a given measure likely to be relevant in athletic performance (Higham et al., 2013; Hopkins, 2004). With this knowledge, athletes can then be guided to perform efficient and adequate warm-up training routines, possibly improving their subsequent performance in power-related tasks. Further studies should be conducted to assess the chronic effects of using optimal individualized PAP protocols on strength, power, and speed performance.

\section{References}

Batterham AM, Hopkins WG. Making meaningful inferences about magnitudes. Int J Sports Physiol Perform, 2006; 1: 50-57

Bevan HR, Cunningham DJ, Tooley EP, Owen NJ, Cook CJ, Kilduff LP. Influence of postactivation potentiation on sprinting performance in professional rugby players. J Strength Cond Res, 2010; 24: 701705

Brown LE, Weir JP. ASEP Procedures Recommendation I: Accurate Assessment of Muscular Strength and Power. J Exerc Physiol, 2001; 4: 1-21

Chatzopoulos DE, Michailidis CJ, Giannakos AK, Alexiou KC, Patikas DA, Antonopoulos CB, Kotzamanidis $\mathrm{CM}$. Postactivation potentiation effects after heavy resistance exercise on running speed. J Strength Cond Res, 2007; 21: 1278-1281

Chiu LZ, Fry AC, Weiss LW, Schilling BK, Brown LE, Smith SL. Postactivation potentiation response in athletic and recreationally trained individuals. J Strength Cond Res, 2003; 17: 671-677

Cormie P, Mcguigan MR, Newton RU. Developing maximal neuromuscular power: part 2 - training considerations for improving maximal power production. Sports Med, 2011a; 41: 125-146

Cormie P, Mcguigan MR, Newton RU. Developing maximal neuromuscular power: Part 1-biological basis of maximal power production. Sports Med, 2011b; 41: 17-38

Crewther BT, Kilduff LP, Cook CJ, Middleton MK, Bunce PJ, Yang GZ. The acute potentiating effects of back squats on athlete performance. J Strength Cond Res, 2011; 25: 3319-3325

Cronin J, Sleivert G. Challenges in understanding the influence of maximal power training on improving athletic performance. Sports Med, 2005; 35: 213-234

Ebben WP. A brief review of concurrent activation potentiation: theoretical and practical constructs. $J$ Strength Cond Res, 2006; 20: 985-991

Esformes JI, Keenan M, Moody J, Bampouras TM. Effect of different types of conditioning contraction on upper body postactivation potentiation. J Strength Cond Res, 2011; 25: 143-148

Fukutani A, Takei S, Hirata K, Miyamoto N, Kanehisa H, Kawakami Y. Influence of the intensity of squat exercises on the subsequent jump performance. J Strength Cond Res, 2014; 28: 2236-2243

Golas A, Maszczyk A, Zajac A, Mikolajec K, Stastny P. Optimizing post activation potentiation for explosive activities in competitive sports. J Hum Kinet, 2016; 52: 95-106

Golas A, Wilk M, Statsny P, Maszczyk A, Pajerska K, Zajac A. Optimizing Half Squat Post Activation Potential Load In Squat Jump Training For Eliciting Relative Maximal Power In Ski Jumpers. J Strength Cond Res, 2017; In Press

Gourgoulis V, Aggeloussis N, Kasimatis P, Mavromatis G, Garas A. Effect of a submaximal half-squats warm-up program on vertical jumping ability. J Strength Cond Res, 2003; 17: 342-344 
Hanson ED, Leigh S, Mynark RG. Acute effects of heavy- and light-load squat exercise on the kinetic measures of vertical jumping. J Strength Cond Res, 2007; 21: 1012-1017

Higham DG, Pyne DB, Anson JM, Eddy A. Physiological, anthropometric, and performance characteristics of rugby sevens players. Int J Sports Physiol Perform, 2013; 8: 19-27

Hodgson M, Docherty D, Robbins D. Post-activation potentiation: underlying physiology and implications for motor performance. Sports Med, 2005; 35: 585-595

Hopkins WG. How to interpret changes in an athletic performance test. Sportsci, 2004; 8: 1-7

Hopkins WG, Marshall SW, Batterham AM, Hanin J. Progressive statistics for studies in sports medicine and exercise science. Med Sci Sports Exerc, 2009; 41: 3-13

Jones $\mathrm{P}$, Lees A. A biomechanical analysis of the acute effects of complex training using lower limb exercises. J Strength Cond Res, 2003; 17: 694-700

Lim JJ, Kong PW. Effects of isometric and dynamic postactivation potentiation protocols on maximal sprint performance. J Strength Cond Res, 2013; 27: 2730-2736

Linder EE, Prins JH, Murata NM, Derenne C, Morgan CF, Solomon JR. Effects of preload 4 repetition maximum on 100-m sprint times in collegiate women. J Strength Cond Res, 2010; 24: 1184-1190

Lowery RP, Duncan NM, Loenneke JP, Sikorski EM, Naimo MA, Brown LE, Wilson FG, Wilson JM. The effects of potentiating stimuli intensity under varying rest periods on vertical jump performance and power. J Strength Cond Res, 2012; 26: 3320-3325

Mola JN, Bruce-Low SS, Burnet SJ. Optimal recovery time for postactivation potentiation in professional soccer players. J Strength Cond Res, 2014; 28: 1529-1537

Rassier DE, Macintosh BR. Coexistence of potentiation and fatigue in skeletal muscle. Braz J Med Biol Res, 2000; 33: 499-508

Ruben RM, Molinari MA, Bibbee CA, Childress MA, Harman MS, Reed KP, Haff GG. The acute effects of an ascending squat protocol on performance during horizontal plyometric jumps.J Strength Cond Res, 2010; 24: 358-369

Sale DG. Postactivation potentiation: role in human performance. Exerc Sport Sci Rev, 2002; 30: 138-143

Scott SL, Docherty D. Acute effects of heavy preloading on vertical and horizontal jump performance. $J$ Strength Cond Res, 2004; 18: 201-205

Seitz LB, De Villarreal ES, Haff GG. The temporal profile of postactivation potentiation is related to strength level. J Strength Cond Res, 2014; 28: 706-715

Smilios I, Pilianidis T, Sotiropoulos K, Antonakis M, Tokmakidis SP. Short-term effects of selected exercise and load in contrast training on vertical jump performance. J Strength Cond Res, 2005; 19: 135-139

Tillin NA, Bishop D. Factors modulating post-activation potentiation and its effect on performance of subsequent explosive activities. Sports Med, 2009; 39: 147-166

Wilson JM, Duncan NM, Marin PJ, Brown LE, Loenneke JP, Wilson SM, Jo E, Lowery RP, Ugrinowitsch C. Meta-analysis of postactivation potentiation and power: effects of conditioning activity, volume, gender, rest periods, and training status. J Strength Cond Res, 2013; 27: 854-859

Young WB, Jenner A, Griffiths K. Acute Enhancement of Power Performance From Heavy Load Squats. J Strength Cond Res, 1998; 12: 82-84

\section{Corresponding author:}

\section{Irineu Loturco}

Nucleus of High Performance in Sport - São Paulo

Av. Padre José Maria, 555 - 04753-060 - São Paulo - SP - Brazil

e-mail: irineu.loturco@terra.com.br 